\title{
Black Hole Spin Down in GRB observations and cosmology
}

\author{
Antonios Nathanail* \\ Research Center for Astronomy and Applied Mathematics, \\ Academy of Athens, Athens 11527, Greece \\ and \\ Section of Astrophysics, Astronomy and Mechanics, Department of Physics, \\ University of Athens, Panepistimiopolis Zografos, Athens 15783, Greece \\ E-mail: antonionitonidhotmail.com
}

\section{Ioannis Contopoulos}

Research Center for Astronomy and Applied Mathematics, Academy of Athens, Athens 11527, Greece

E-mail: icontopdacademyofathens.ar

\section{Spyros Basilakos}

Research Center for Astronomy and Applied Mathematics,

Academy of Athens, Athens 11527, Greece

E-mail: Svasileacademyofathens.gr

According to Blandford \& Znajek (1977), energy can be extracted electromagnetically from a rotating black hole, should the latter be endowed with a magnetic field supported by electric currents in a surrounding disk. We show that exact models of black hole magnetospheres produce Poynting flux that decreases almost exponentially with time. We went through the Swift BATXRT lightcurves and identified a subclass of GRBs that exhibits a clear exponential decay over more than three orders of magnitude in flux (EDOHS GRBs). We estimate the energy given-off in the X-rays and discuss a possible correlation between the peak brightness of the X-ray prompt emission and its decay time. We investigate a possible application of this result in high redshift Cosmology.

Swift: 10 Years of Discovery,

2-5 December 2014

La Sapienza University, Rome, Italy

\footnotetext{
* Speaker.
} 


\section{Introduction}

Gamma-ray bursts (hereafter GRBs) have been a great scientific puzzle since their discovery in the $60 \mathrm{~s}$. For more than 20 years, the only information we had about them was that they produced flashes of gamma-rays. Their isotropic distribution in the sky suggested a cosmological origin , and this was confirmed with the detection of the afterglow in optical and other wavelengths that allowed the determination of their redshift. It has been widely discussed that GRBs fall into two subcategories so-called short- and long-duration, which are believed to be associated with neutron star-neutron star mergers and black hole formation during super-massive star core collapse respectively.

A great amount of theoretical work has been invested in order to understand what is the central engine and the emission mechanism of GRBs. Several researchers believe that the central engine is a black hole (for example [四). In recent years the question of the central engine has been put aside, while research focuses on the emission region, the emission mechanisms and the effort to understand all the characteristics of the light curves and the spectra of the bursts (for a recent review []] ). The idea of a black hole powering the burst is widely accepted for the so-called long-duration GRBs, where the source of the burst is believed to be the electromagnetic extraction of energy from the black hole rotation ([B]]). Obviously, as the black hole loses energy, it will spin down. The question is at what rate. [四] calculated in detail the black hole electromagnetic spindown rate and showed that the extracted Poynting flux decreases almost exponentially with time (see next section).

GRBs may also be of fundamental importance for Cosmology since they are observed up to very high redshifts at which the distance modulus is most sensitive to the cosmological parameters. This makes them ideal potential tracers of the Hubble relation if we could somehow associate their absolute luminosity with one (or more) of their observable parameters. Several authors have tested the cosmological implications of GRBs through several empirical correlations between various properties of the prompt and in some cases also the afterglow emission, and their results have been eagerly applied to constrain cosmological parameters .

In the present work we went through the Swift BAT/XRT data in order to find a clear exponential decay of energy flux in time since the trigger of the burst, which would indicate that we may be following a black hole spinning down. We restrict ourselves to GRBs with known redshift, so that we can estimate the energetics of each burst. We identify a certain subclass of 14 long duration GRB events which follow a certain correlation between the peak brightness of the X-ray prompt emission and its decay time. We discuss how this correlation may be used in high redshift cosmology.

\section{Black Hole Electromagnetic Spin Down}

The extraction of the rorational energy of a black hole through the Blandford \& Znajek (1977) mechanism is widely accepted and discussed in the literature. As we said, extraction of rotational energy from the black hole actually corresponds to black hole spin down. We review here the main elements of black hole spin down as obtained in detail in references [四]. Let us consider a supermassive star whose core collapses and forms a maximally rotating black hole. If the star is 
magnetized, magnetic flux will be advected with the collapse. The material that is going to collapse into a black hole will be strongly magnetized, and therefore its core will pass through a spinning magnetized neutron star stage. A certain amount of magnetic flux $\Psi_{m}$ is then going to cross the horizon. An equatorial thick disk will form around the black hole due to the rotational collapse. This material will hold the magnetic flux advected initially toward the horizon and (at least for a limited time) will prevent it from escaping to infinity. As long as this is the case, the black hole will lose rotational/reducible energy at a rate

$$
\dot{E} \approx-\frac{1}{6 \pi^{2} c} \Psi_{m}^{2} \Omega^{2}
$$

and will thus spin down very dramatically ([3]) for low spin parameters; references in [[]], [目] for maximally rotating black holes). Moreover, some (yet unknown) fraction $f_{\mathrm{X}}$ of it will be observed in X-rays, namely $\dot{E}_{\mathrm{X}} \sim f_{\mathrm{X}} \dot{E}$.

A note on the production of high energy radiation in the black hole magnetosphere is in order here. We have found that a generic feature of black hole magnetospheres is a poloidal electric current sheet that originates on the horizon at the equator. Our observational and theoretical experience from pulsars suggests that high energy radiation is expected to originate from reconnection

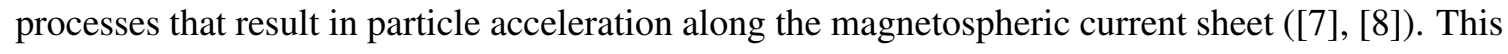
implies that high energy radiation may not be coming along the axis of rotation but in a direction orthogonal to it. Obviously, further out, the current sheet and the consequent high-energy radiation will be naturally collimated along the axis of rotation by the stellar material surrounding the black hole.

Let us now return to the calculation of the black hole spin down. The available rotational/ reducible black hole energy is $a^{\mathscr{G}} M^{2} \Omega / c$ where $a \equiv J / M$ is the black hole spin parameter, and $\mathscr{G}$ is the gravitational constant. The black hole will therefore spin down as $\dot{E}=\frac{\mathscr{G} M^{2}}{c} \frac{\mathrm{d}(a \Omega / M)}{\mathrm{d} t}$, Equating this with eq. (2. (1) we find an approximate solution of the rate of the black hole spin down (the exact solution can be found in [四])

$$
\dot{E} \approx \dot{E}_{o} e^{-t / \tau}
$$

where $\tau \equiv \frac{24 c^{5}}{\mathscr{G}^{2} B^{2} M}=7\left(\frac{B}{10^{16} \mathrm{G}}\right)^{-2}\left(\frac{M}{M_{\odot}}\right)^{-1}$ sec is a very important physical parameter of the model. Here, $B$ is the initial black hole magnetic field. As we will discuss below, $\tau$ may be directly observable.

One can directly check that, for a magnetic field $>10^{15} \mathrm{G}$, the black hole spins down in a few tens to a few hundred seconds. Obviously, such ultra-strong magnetic fields can survive only during the core-collapse of a massive star, and are subsequently dispersed away from the black hole horizon. We are thus willing to make a tentative association between GRB events where eq. ([2.2) yields a good fit in the decay of the prompt emission and the electromagnetic spin down of a newly formed maximally rotating stellar mass black hole.

\section{GRBs with Exponential Decay in One Hundred Seconds}

GRB prompt (and afterglow) emission light curves are in general rather complicated with multi peak sub-structure. We want to find signs of black hole spindown in GRB observations, that is why 


$\begin{array}{lcccc}\text { Name } & z & F_{\mathrm{obs}}\left( \pm \sigma_{F_{\mathrm{obs}}}\right) & \tau_{\mathrm{obs}}\left( \pm \sigma_{\tau_{\mathrm{obs}}}\right) & E_{X}\left( \pm \sigma_{E_{X}}\right) \\ & & 10^{-8} \frac{\mathrm{erg}}{\mathrm{s} \mathrm{cm}^{2}} & \mathrm{~s} & 10^{51} \mathrm{erg} \\ & & & & \\ \text { 130831A } & 0.4791 & 21( \pm 10) & 8.5( \pm 1) & 1.26( \pm 0.45) \\ \text { 100418A } & 0.6235 & 8( \pm 1) & 6( \pm 1) & 0.33( \pm 0.07) \\ \text { 080916 } & 0.689 & 7( \pm 1.2) & 10.1( \pm 2) & 0.83( \pm 0.22) \\ \text { 061110A } & 0.757 & 4.6( \pm 2) & 10.1( \pm 1.3) & 1.06( \pm 0.48) \\ \text { 080430 } & 0.767 & 10.6( \pm 2) & 5.2( \pm 2) & 2.3( \pm 0.98) \\ \text { 070714B } & 0.92 & 5.4( \pm 2) & 29( \pm 2) & 3.68( \pm 1.39) \\ \text { 051006 } & 1.059 & 6.56( \pm 1.4) & 7.5( \pm 0.7) & 1.31( \pm 0.3) \\ \text { 110808A } & 1.348 & 2.36( \pm 1) & 11( \pm 0.7) & 1.13( \pm 0.48) \\ \text { 120724A } & 1.48 & 2.1( \pm 0.7) & 17( \pm 1.4) & \\ \text { 060708 } & 1.92 & 4( \pm 0.8) & 7( \pm 0.8) & \\ \text { 070110 } & 2.352 & 2( \pm 0.9) & 21.4( \pm 2.1) & \\ \text { 111107A } & 2.893 & 0.5( \pm 0.3) & 27.5( \pm 3.5) & \\ \text { 091109A } & 3.076 & 2.4( \pm 1.2) & 23.5( \pm 3.1) & \\ \text { 090519 } & 3.85 & 1.7( \pm 0.5) & 39.4( \pm 4.5) & \end{array}$

Table 1: EDOHS GRB Observations, 8 GRBs with $z<1.4$ in the range of available SnIa data, 2 GRBs with intermediate redshifts $1.4<z \leq 2$ and 4 GRBs with high redshift $z>2$.

we decided to focus only on GRBs with a single exponentially decaying prompt emission event. Furthermore, we wanted to find similar systems so as to explore and compare the energetics of the bursts. We thus formulated the following empirical criteria that characterize a particular subclass of gamma-ray bursts: 1) A single prompt emission event in 15 to $50 \mathrm{keV} \mathrm{X-rays;} \mathrm{2)} \mathrm{Exponential}$ prompt emission decay over more than three orders of magnitude; 3) Prompt emission duration (up to the first break where the energy flux seems to be constant) longer than about 100 seconds, but not much longer than a few hundred seconds (ad hoc value); 4) Full (not sparse) sampling of the light curve during that time interval; 5) Known redshift. This is required in order for us to test our model of standard explosions for Cosmology.

The new GRB sub-class defined by the above criteria (hereafter EDOHS GRBs) has similarities with previously defined sub-classes such as Fast Rise Exponential Decay-FREDs selected by the Compton GRO collaboration ([Q] $)$. We worked with the Swift data and went through all the Swift-BAT/XRT light curves from 2005 till 2014 ([ए]] , [प])). We found 14 GRBs that fulfil our criteria and their BAT+XRT light curves can be fit with a simple exponential (note that such a fit can be discerned more clearly in a log-linear plot; see Appendix). For each one of these events, the fit gave us the observed characteristic exponential decay timescale $\tau_{\mathrm{obs}}$ and the peak observed flux $F_{\text {obs }}($ Table 1).

The signs of exponential decay in our sample allow us to believe that we may actually be observing the electromagnetic spin down of the newly formed black hole. Furthermore, the deduced decay times show a clear statistical time dilation with redshift (with only a couple notable exceptions; see Fig. 1), which suggests that they may indeed consist an unbiased representation of their intrinsic values. Notice that this is not the case for the larger GRB population where sev- 

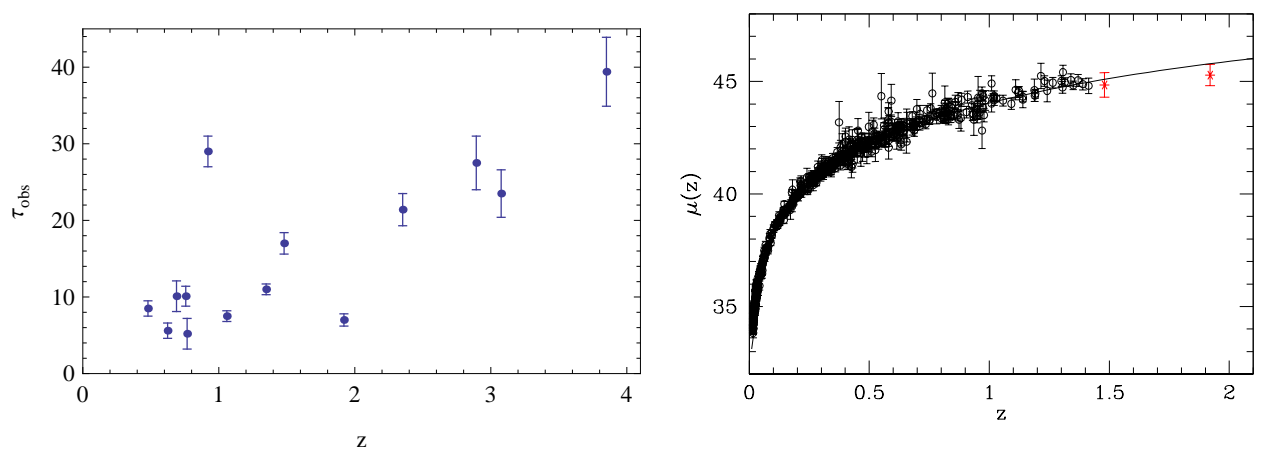

Figure 1: Left:The observational characteristic exponential decay timescale $\tau_{o b s}$ versus redshift $z$, signs of time dilation are evident in this plot. Right:Comparison between the estimated distance modulus for the intermediate EDOHS GRB observations $(1.4<z \leq 2$, red stars $)$ and the recent SnIa data-set of [ए]] $\left(0.015 \leq z \leq 1.414\right.$, circles). The solid curve corresponds to the concordance $\Lambda$ CDM model with $\Omega_{m 0}=0.30$ and $H_{0}=70 / \mathrm{km} / \mathrm{s} / \mathrm{Mpc}$.

eral observational selection biases obscure the cosmological time dilation. In other words there is some indication that our selected GRB subclass (but not the entire GRB population) may consist alternative cosmological distance indicators.

In the case of exponential decay that we are currently investigating, the total energy $E_{\mathrm{X}}$ givenoff in X-rays can be estimated as $E_{\mathrm{X}}=\frac{4 \pi}{1+z} d_{\mathrm{L}}^{2}(z) F_{\mathrm{obs}} \tau_{\mathrm{obs}}$, where, $d_{\mathrm{L}}$ is the luminosity distance. We acknowledge that our estimation is rather crude, and plan to study the spectra in a future work.

We can estimate the luminosity distance of EDOHS GRBs in the SnIa range $(0<z<1.4)$ and calculate the energy $E_{\mathrm{X}}$ given-off in the X-rays. We tested whether $E_{\mathrm{X}}$ depends on redshift. We find no significant correlation between $E_{\mathrm{X}}$ and redshift in that range (a $\chi^{2}$-minimization in $\log E_{\mathrm{X}}-$ $\log (1+z)$ space yields an unacceptably high reduced chi-square fit, $\chi_{v}^{2} \gg 6.8$ and the probability to fit the data is $\left.\sim 10^{-7}\right)$. The total X-ray energy release is $E_{\mathrm{X}} \simeq 1.49( \pm 0.55) \times 10^{51} \mathrm{erg}$.

\section{Discussion and conclusions}

In our present study, we analyzed Swift-XRT/BAT light curves (2005-2014) to find GRBs that fulfill a set of phenomenological criteria defined in $\S 3$. In particular, we did a systematic search for clear signs of exponential decay in the prompt and afterglow emission, and obtained a new subclass of EDOHS GRBs. We tentatively associated EDOHS GRBs with the electromagnetic spin down of the black hole that forms during the core collapse of a massive star. If that is indeed the case, then we may be probing the Blandford-Znajek process in action.

We propose to do high redshift cosmology with EDOHS GRBs. Unfortunately, beyond redshift $z \approx 2$ we are reaching the Swift-BAT trigger limit for EDOHS, thus our current high redshift events are not reliable cosmological tracers. Future detectors will solve this problem.

This work made use of data supplied by the UK Swift Science Data Centre at the University of Leicester, and was supported by the General Secretariat for Research and Technology of Greece and the European Social Fund in the framework of Action 'Excellence'. 

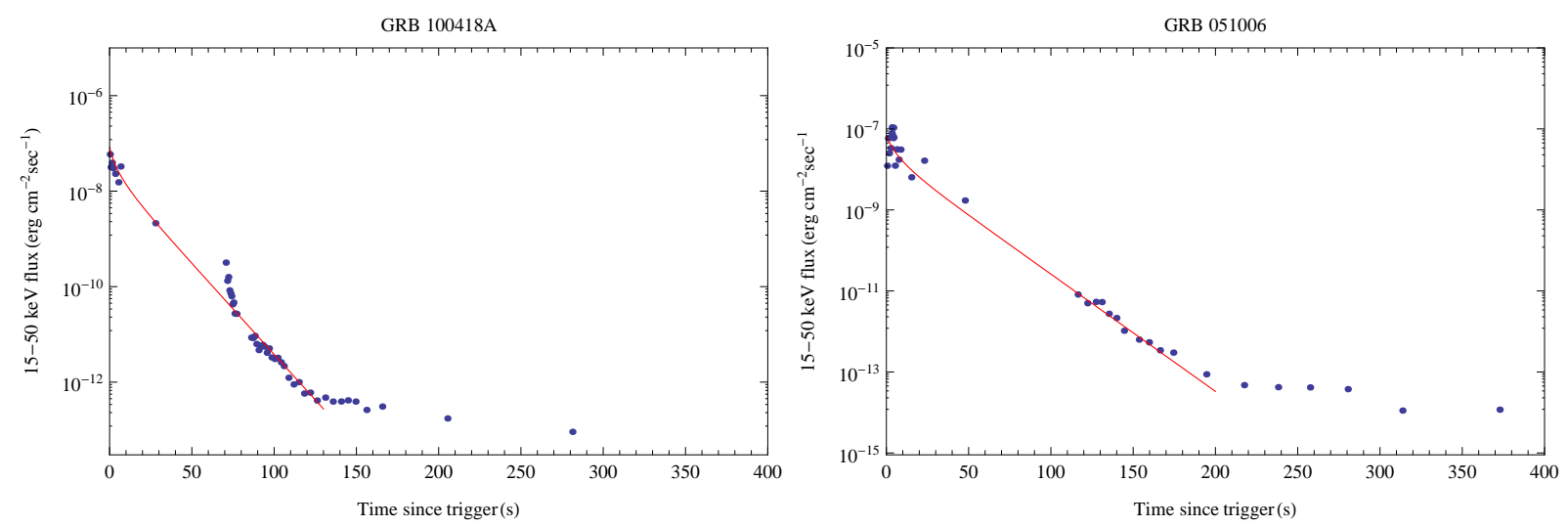

Figure 2: Two of the selected GRBs with Exponential Decay in One Hundred Seconds - EDOHS with best fits of their prompt emission almost exponential decay. The fits yield the values of $\tau_{\mathrm{obs}}$ and $F_{\mathrm{obs}}$ shown in Table 1.

\section{References}

[1] S. E. Woosley, Gamma-ray bursts from stellar mass accretion disks around black holes, ApJ 405 (1993) 273

[2] P. Kumar, \& B. Zhang, The Physics of Gamma-Ray Bursts and Relativistic Jets, (2014) arXiv:1410.0679

[3] R. D. Blandford, \& R. L. Znajek, Electromagnetic extraction of energy from Kerr black holes, MNRAS 179 (1977) 433

[4] I. Contopoulos, A. Nathanail \& D. Pugliese, The Orthogonal Gamma-Ray Burst Model, ApJ 780 (2014) 5

[5] I. Contopoulos, D. Kazanas \& D. B. Papadopoulos, The Force-free Magnetosphere of a Rotating Black Hole, ApJ 765 (2013) 113

[6] A. Nathanail \& I. Contopoulos, Black Hole Magnetospheres, ApJ 788 (2014) 186

[7] C. Kalapotharakos, A. K. Harding, D. Kazanas \& I. Contopoulos, Gamma-Ray Light Curves from Pulsar Magnetospheres with Finite Conductivity, ApJ 754 (2012) 1

[8] L. Sironi \& A. Spitkovsky, Relativistic Reconnection: An Efficient Source of Non-thermal Particles, ApJ 783 (2014) 21

[9] D. Kocevski, F. Ryde \& E. Liang, Search for Relativistic Curvature Effects in Gamma-Ray Burst Pulses, ApJ 596 (2003) 389

[10] P. A. Evans et al. An online repository of Swift/XRT light curves of gamma-ray burstsl, A\& A 469 (2007) 379

[11] P. A. Evans et al. Methods and results of an automatic analysis of a complete sample of Swift-XRT observations of GRBs, MNRAS 397 (2009) 1177

[12] N. Suzuki et al. The Hubble Space Telescope Cluster Supernova Survey. V. Improving the Dark-energy Constraints above z $>1$ and Building an Early-type-hosted Supernova Sample, ApJ 746 (2012) 85 